\title{
A CANDIDATE SUBSTELLAR COMPANION TO HR 7329
}

\author{
Patrick J. Lowrance, ${ }^{1}$ Glenn Schneider, ${ }^{2}$ J. Davy KirkPatrick, ${ }^{3}$ E. E. BeCKlin, ${ }^{1}$ Alycia J. Weinberger, ${ }^{1}$ \\ B. Zuckerman, ${ }^{1}$ Phil Plait, ${ }^{4}$ Eliot M. Malmuth, ${ }^{5}$ Sara R. Heap, ${ }^{6}$ A. Schultz, ${ }^{7}$ Bradford A. Smith, ${ }^{8}$ Richard \\ J. TeRrile, ${ }^{9}$ AND DEAN C. HINeS ${ }^{2}$ \\ Received 2000 January 24; accepted 2000 May 8
}

\begin{abstract}
We present the discovery of a candidate substellar companion from a survey of nearby young stars made with the Near-Infrared Camera and Multiobject Spectrometer coronagraph on the Hubble Space Telescope. The $H \approx 12 \mathrm{mag}$ object was discovered approximately 4" from the young A0 V star HR 7329 . Using follow-up spectroscopy from the Space Telescope Imaging Spectrograph, we derive a spectral type between M7 V and M8 V with an effective temperature of $\sim 2600 \mathrm{~K}$. We estimate that the probability of a chance alignment with a foreground dwarf star of this nature is $\sim 10^{-8}$, and therefore we suggest that the object (HR 7329B) is physically associated with HR 7329, with a projected separation of 200 AU. Current brown dwarf cooling models indicate a mass of less than $50 M_{\text {Jup }}$ for HR 7329B based on age estimates of $\leq 30 \mathrm{Myr}$ for HR 7329A.
\end{abstract}

Subject heading: stars: low-mass, brown dwarfs

\section{INTRODUCTION}

The discovery of substellar objects in stellar systems is a key goal in contemporary astronomy and an essential element in furthering our knowledge of the mass function of binary star and planetary system formation. The substellar mass range from 10 to $80 M_{\text {Jup }}\left(0.01-0.08 M_{\odot}\right)$ is crucial to our understanding of the bridge between the lowest mass stars and the giant planets. To this end, the Near-Infrared Camera and Multiobject Spectrometer (NICMOS) Instrument Definition Team (IDT) has conducted an infrared coronagraphic survey of young main-sequence stars in order to search for substellar companions. Substellar objects cool with age because they do not sustain hydrogen fusion and are more difficult to detect with time because they become fainter (e.g., Burrows et al. 1997). Using independently determined ages and distances for the target stars, the masses of newly detected secondaries can be ascertained from infrared fluxes and theoretical evolutionary tracks on the H-R diagram. Follow-up spectroscopy further constrains the effective temperature and probability of companionship. Here we present a spectrum obtained with the Space Telescope Imaging Spectrograph (STIS) of a substellar companion candidate, HR 7329B, from our NICMOS imaging survey. Previously, this survey revealed TWA 5B, an $\sim 20 M_{\text {Jup }}$ brown dwarf companion to TWA 5A (Lowrance et al. 1999).

\footnotetext{
${ }^{1}$ Department of Physics and Astronomy, University of California at Los Angeles, 405 Hilgard Avenue, Los Angeles, CA 90095.

${ }^{2}$ Department of Astronomy and Steward Observatory, 933 North Cherry Avenue, University of Arizona, Tucson, AZ 87521.

${ }^{3}$ Infrared Processing and Analysis Center, California Institute of Technology, 770 South Wilson Avenue, Pasadena, CA 91125.

4 Advanced Computer Concepts, Inc., Goddard Space Flight Center, Greenbelt, MD 20771.

${ }^{5}$ Raytheon ITSS, Goddard Space Flight Center, Greenbelt, MD 20771.

${ }^{6}$ Goddard Space Flight Center, Greenbelt, MD 20771.

${ }^{7}$ Computer Sciences Corporation, 7700 Hubble Drive, LanhamSeabrook, MD 20706.

${ }^{8}$ Institute of Astronomy, University of Hawaii, 2680 Woodlawn Drive, Honolulu, HI 96822.

${ }^{9}$ Jet Propulsion Laboratory, California Institute of Technology, 4800 Oak Grove Drive, Pasadena, CA 91109.
}

\section{NICMOS}

\subsection{Observations}

HR 7329 [HD 181296; A0 V; $d \sim 48$ pc; $V=5.05$; R.A. $=19^{\mathrm{h}} 22^{\mathrm{m}} 51^{\mathrm{s}} .2, \quad$ Decl. $\left.=-54^{\circ} 25^{\prime} 26^{\prime \prime} \quad(\mathrm{J} 2000.0)\right]$ was observed with NICMOS on 1998 June 29 from 16:15 to 17:17 UT. We obtained multiple-exposure images with the star behind the coronagraph (radius $=0.3$ ) on camera 2 (pixelscale $=\sim 0$ 0.076 pixel $^{-1}$ ) and a wide-band F160W filter (central wavelength $=1.5940 \mu \mathrm{m}, \Delta \lambda=0.4030 \mu \mathrm{m}$ ), which corresponds closely to the central wavelength of a Johnson $H$-band photometric filter. Five standard NICMOS STEP16 MULTIACCUM (nondestructive read) integrations (MacKenty et al. 1997) totaling $719.6 \mathrm{~s}$ were executed at each of two orientations differing by $29^{\circ} .9$. While the stellar point-spread function (PSF), the instrumental scattering function, and detector artifacts rotate with the aperture, any real features in the unocculted area of the detector will be unaffected by a change in the camera orientation. Subtraction of these two images has been shown to significantly reduce residual PSF background light (Schneider et al. 1998). The NICMOS coronagraphic images were reduced and processed utilizing calibration darks and flat fields created by the NICMOS IDT from on-orbit observations following the method described in Lowrance et al. (1999).

\subsection{Results}

Subtraction and analysis of the NICMOS coronagraphic images reveal a stellar-like object (HR 7329B) at a separation of 4 ". $17 \pm 0.05$ and a position angle of $166.8 \pm 0.2$ from HR 7329 (HR 7329A) (Fig. 1). This secondary is pointlike with an FWHM of 0 "'15 (the diffraction limit is 0 "14), with the first Airy ring apparent in Figure 1. Since the target star is occulted in the NICMOS coronagraphic images, its position is ascertained from the target acquisition image and located behind the coronagraph by a known telescope offset.

The secondary fell near the edge of the field of view in the second orientation, so the magnitude of HR 7329B was measured using a 12 pixel radius circular aperture centered on the companion in the subtracted image from the first 


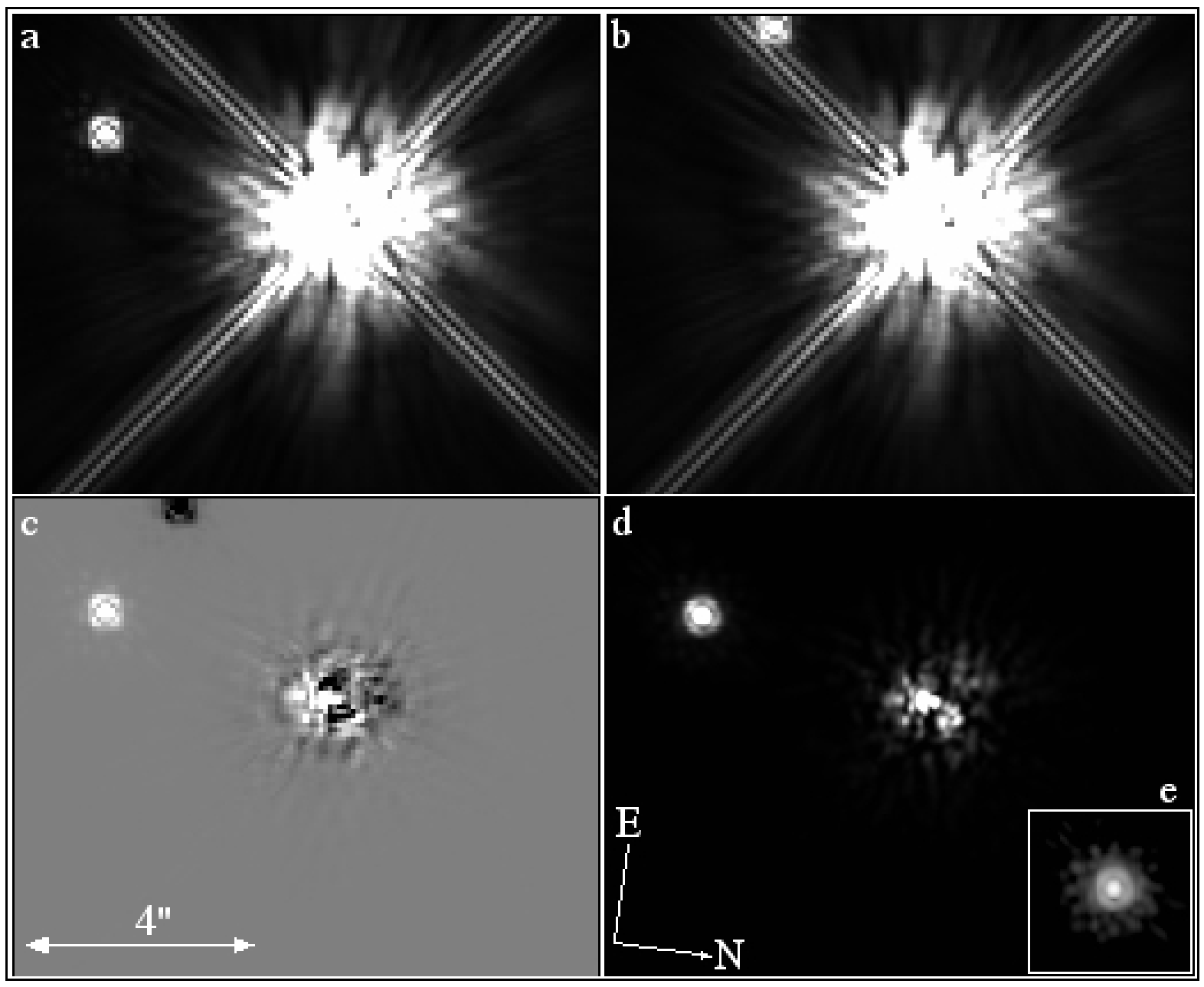

FIG. 1.-NICMOS H-band image of HR 7329B. Observations at two different roll orientations $(a, b)$ have been subtracted with HR 7329A behind the coronagraph $(c)$. The diffraction spikes were masked, and the images were rolled about the primary center and co-added $(d)$, leaving a subsampled image $(e)$ of HR 7329B.

orientation. A correction factor of $9.66 \%$, determined from coronagraphic photometric aperture corrections developed by the NICMOS IDT, was applied to compensate for the flux that fell out of this aperture. The $[\mathrm{F} 160 \mathrm{~W}]$ magnitude of $\mathrm{HR} 7329 \mathrm{~B}$ is then $11.90 \pm 0.06 \mathrm{mag}$ using a conversion factor for the F160W filter of $2.19 \times 10^{-6} \mathrm{Jy} \mathrm{ADU}^{-1} \mathrm{~s}$, and $1083 \mathrm{Jy}$ corresponding to an $H$ magnitude of zero in the Vega system (M. Rieke 1999, private communication) where the majority of the uncertainty is dominated in NICMOS's calibration in relation to standard stars. The F160W filter is $\sim 30 \%$ wider than the ground-based Johnson $H$-band filters, which necessitates a careful conversion from F160W to $H$ band for cool temperature objects. For six M dwarfs between spectral types M6 and M9 with measured F160W and ground-based $H$-band magnitudes, we find a mean difference of $0.03 \pm 0.02 \mathrm{mag}$. For HR 7329B (M7.5) we thus expect the H-[F160W] color to be about 0.03 mag. Making this color correction, we estimate an $H$ magnitude of $11.93 \pm 0.06 \mathrm{mag}$.

The $[\mathrm{F} 187 \mathrm{~N}]$ magnitude of HR 7329A was determined using the aperture photometry of the two calibrated target acquisition images (at each of the two spacecraft orientations) processed as described in Lowrance et al. (1999). Within the uncertainties, the two measurements agreed and were averaged to yield $[\mathrm{F} 187 N]=5.0 \pm 0.1$ mag.

\section{STIS}

\subsection{Observations}

HR 7329 was acquired in the STIS 52" $\times 0$ 0"2 slit on 1999 May 20 and then offset by 0.95 in right ascension and -4 .06 in declination (based on the NICMOS astrometric results) to place the secondary into the slit. To keep the primary as far out of the slit as possible, we employed a slit position angle of 252.06 so that the line joining the primary and secondary was approximately perpendicular to the slit, thereby minimizing contamination from scattered primary light. Spectral imaging sequences were completed in one orbit with the G750M grating in three tilt settings with central wavelengths of 8311,8825 , and $9336 \AA$ with a resolution of $\sim 0.55 \AA$ for total integration times of 340,172 , and $150 \mathrm{~s}$, respectively. At each tilt setting, we executed a two-position dither of 0.35 along the slit to allow for the replacement of bad or hot pixels, and the exposures were split for cosmic-ray removal. Thus, we obtained four spectra at each tilt setting. After each set of four spectral images, we obtained the flat fields required to calibrate the known 
effects of fringing that appear longward of $\sim 7500 \AA$. Due to a failure of the Hubble Space Telescope to acquire one of the two guide stars, there was a small differential pointing error of about $0^{\prime \prime} 04$, or 1 pixel. This caused the secondary to be marginally decentered, and as a result a small percentage of the target flux fell out of the slit.

\subsection{Results}

The STIS spectra were calibrated, averaged, binned to a resolution of $\sim 6 \AA$, and normalized to the flux (in units of ergs s${ }^{-1} \mathrm{~cm}^{-2} \AA^{-1}$ ) at $8500 \AA$. We compared the final total spectrum with those of standard low-temperature dwarf and giant star spectra with a resolution equal to $18 \AA$, which is a factor of 3 lower than our STIS spectrum (Kirkpatrick, Henry, \& McCarthy 1991; Kirkpatrick, Henry, \& Irwin 1997; see Fig. 2). The HR 7329B spectrum contains an absorption line near $8200 \AA$, which we attribute to the NaI doublet that does not appear in late-type giant stars but is nicely fitted in the dwarfs. Also, as seen in Figure 2, the slope of the spectrum from 8600 to $8850 \AA$ is small, as in the dwarf spectra, whereas it rises sharply for giant stars. The $\mathrm{NaI}$ line is fitted very well by the M8 V standard, but the TiO absorption near $8860 \AA$ is best fitted by the M7 V spectrum. We therefore assign HR 7329B a spectral type of M7.5 V with an uncertainty of 0.5 spectral type.

The diffraction spikes from the primary star also fall in the slit above and below the secondary, and we used the relative positions of the three resulting spectra to determine the primary-secondary separation. The result of 4.13 \pm 0 "'05 agrees within the uncertainties with the NICMOS measured separation reported in $\S 2.2$.

\section{DISCUSSION}

\subsection{Likelihood of Companionship}

From its $\mathrm{H}$-magnitude and M7.5 V spectral type, HR 7329B can be either a background object, a foreground main sequence $M$ star, or a companion to HR 7329A. A main-sequence M7.5 V star has an absolute magnitude $M_{H}=10.3$ (Kirkpatrick \& McCarthy 1994), so HR 7329B is too bright to be a background main-sequence star. If it were on the main-sequence, its photometric distance would be 19 pc. Henry (1991), in a volume-limited infrared survey, finds six objects with an $M_{H}>9.5$ within $5 \mathrm{pc}$ of the Sun. If we assume a spherical distribution of low-mass stars in the solar neighborhood, we can extrapolate the results within 5 pc to expect 1000 such objects within $25 \mathrm{pc}$, so the a priori probability of finding one in projection within a 4 " radius circle is $\sim 10^{-7}$.

Proper-motion measurements of the companion and the primary in the time between the NICMOS and STIS observations could be used to constrain the probability of companionship further. Unfortunately, the positional errors are too large. However, we can further constrain the probability that the object is not a foreground $M$ dwarf. Searching the Tycho catalog, we find that the mean proper motion of 1000 stars between 16 and $25 \mathrm{pc}$ is $0^{\prime \prime} \cdot 373 \pm 0^{\prime \prime} .277$. Therefore, if

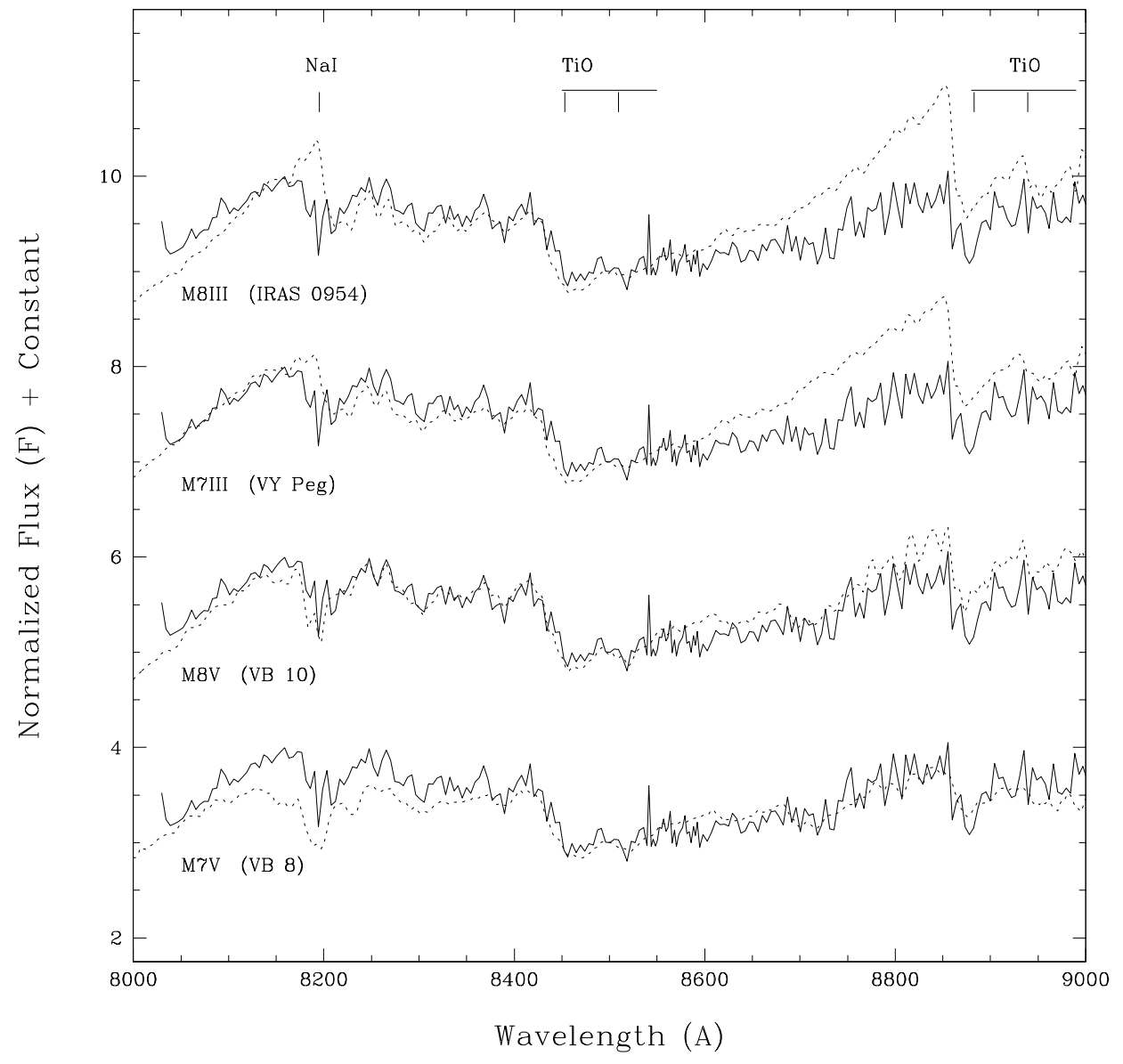

FIG. 2.-STIS spectrum of HR 7329B (solid line) normalized from units of ergs s $\mathrm{s}^{-1} \mathrm{~cm}^{-2} \AA^{-1}$ compared with standard late-type M dwarf and giant spectra (dashed line) (Kirkpatrick at al. 1991, 1997). The zero level of each spectrum is 2, 4, 6 and 8, respectively. The giant spectra fitted neither the NaI absorption near $8200 \AA$ nor the slope later than $8600 \AA$. The best fit lies between M7 V and M8 V (the longward cutoff of $9000 \AA$ is where the signal-to-noise ratio of the STIS spectrum becomes too low because of fringing effects). 
we assume a Gaussian distribution of proper motions about this mean, almost $80 \%$ of foreground stars $\left(0^{\prime \prime} 1\right)$ have moved (taking into account angles along the slit) more than the half-width of the STIS slit and would not be visible in the second epoch. Given these arguments, it is unlikely $\left(\sim 10^{-8}\right)$ that HR $7329 \mathrm{~B}$ is a foreground object, and for the remainder of the paper we assume it is physically associated with HR 7329A.

\subsection{Age of the System}

It is difficult to determine an age for A-type stars, but HR 7329 appears to be young ( $<40 \mathrm{Myr}$ ) based on its rotation and, more importantly, its location on an H-R diagram. For massive stars, rotational velocities decline with age; HR 7329 has an especially large $v \sin i, 330 \mathrm{~km} \mathrm{~s}^{-1}$ (Abt \& Morrel 1995), that is considerably above that of the majority of A-type stars $\left(\sim 100 \mathrm{~km} \mathrm{~s}^{-1}\right)$. Figure 3 reproduces the H-R diagram from Jura et al. (1998) for A stars from the Yale Bright Star Catalog and overplots nearby young clusters. There seem to be common areas of similar age stars; the 50-90 Myr IC 2391 and $\alpha$ Per clusters lie below the older (600 Myr) Hyades and Preasepe. There is a large scatter in the Pleaides (70-125 Myr), which could be due to a range of distances and ages as well as unresolved binaries. HR 7329 lies on a line located below the $\alpha$ Per and the IC 2391 cluster that intersects $\beta$ Pic, HR 4796, and HD 141569. The latter stars have recently been assigned ages from their late-type companions of 20,8 , and $5 \mathrm{Myr}$, respectively (Barrado y Navascues et al. 1999; Stauffer, Hartmann, \& Barrado y Navascues 1995; Weinberger et al. 2000). This suggests that HR 7329 is between 10 and $30 \mathrm{Myr}$ old. Finally, it has recently been suggested that HR 7329 is found within a young comoving cluster much like the TW Hydrae Association with an age of $~ 40 \mathrm{Myr}$ (Zuckerman \& Webb 2000; R. A. Webb et al. 2000, in preparation).

\subsection{Effective Temperature and Bolometric Luminosity}

An effective temperature for HR 7329B is required to position it on an H-R diagram, but the temperature scale for late young M dwarfs is uncertain (Allard et al. 1997). Luhman \& Rieke (1998) extrapolate from the model fits of Leggett et al. (1996) to derive 2670 and $2505 \mathrm{~K}$ for M7 V and M8 V, respectively, which agree with the newer models used by Leggett, Allard, \& Hauschildt (1998) with an uncertainty of about $100 \mathrm{~K}$. With this uncertainty for late-M dwarf stars and the added uncertainty due to the spectral type, we plot the derived temperatures for each spectral class and their associated uncertainty (Fig. 4), which overlaps and gives a possible range from 2405 to $2770 \mathrm{~K}$.

The parallactic distance measured to HR 7329A by the Hipparcos mission is $47.67 \pm 1.6 \mathrm{pc}$. With a derived $H$ magnitude of 11.93 for HR 7329B and a distance modulus of 3.39 , we calculate an absolute $\mathrm{H}$ magnitude of $8.54 \mathrm{mag}$. There exists a number of bolometric corrections (BCs) in

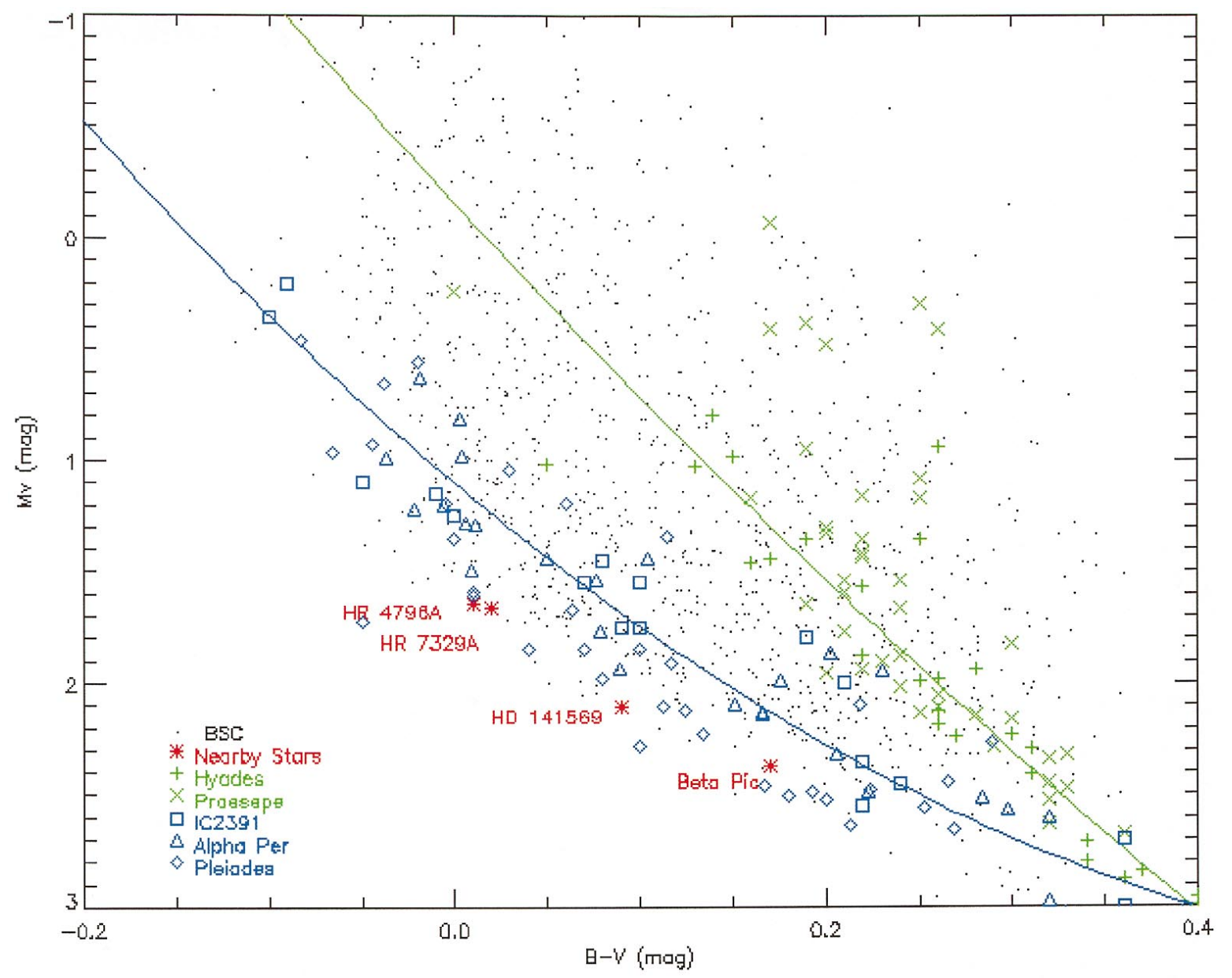

FIG. 3.-H-R diagram for A-type stars in the Yale Bright Star Catalog reproduced from Jura et al. (1998) with the nearest star clusters plotted. The lines indicate common centers for Hyades/Preasepe (600 Myr) and $\alpha$ Per/IC 2391 (50-90 Myr). The asterisks indicate HR 7329 and the nearby stars assigned young ages (4-20 Myr) from late-type companions. 


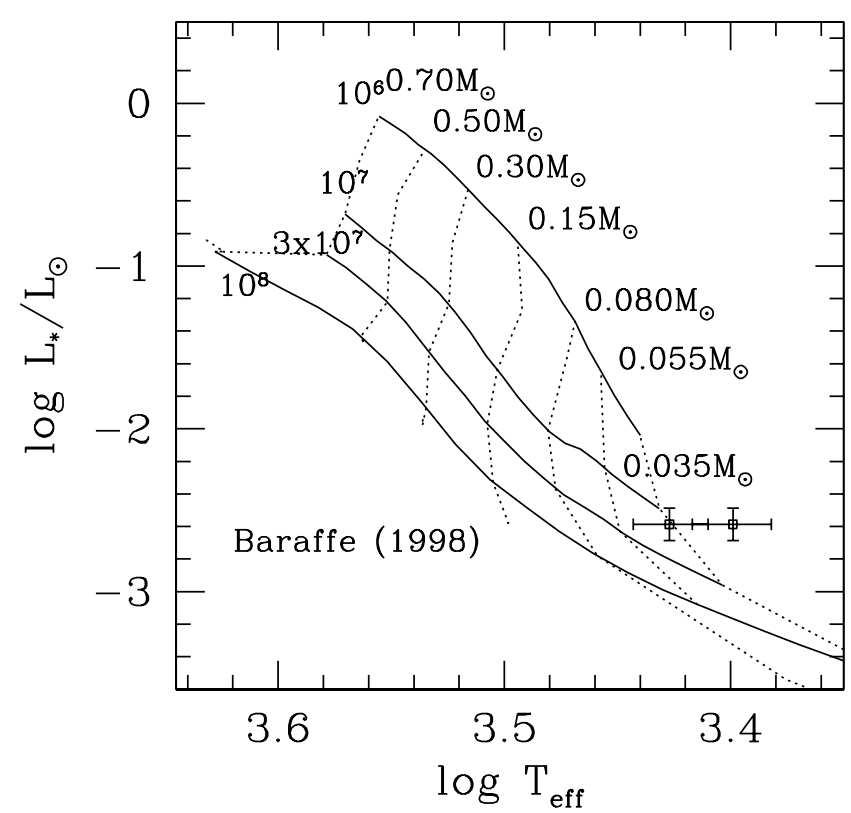

Fig. 4.-Evolutionary tracks (Baraffe et al. 1998) with HR 7329B (squares) plotted at the derived temperatures for M7 V and M8 V with the uncertainty (which overlap) in assigning a temperature to a low-mass star. From these and other evolutionary models, we derive an age of less than 30 Myr for this pair and a mass of less than $50 M_{\text {Jup }}$ for the secondary.

the literature for M7 V and M8 V stars (Tinney, Mould, \& Reid 1993; Kirkpatrick et al. 1993; Bessel, Castelli, \& Plez 1998 ) based on $I$ and $K$ magnitudes. However, none give the $\mathrm{BC}$ in the $H$ band. We have used the $\mathrm{BC}$ at the other bandpasses and the colors of late-type stars as a function of spectral type from Kirkpatrick \& McCarthy (1994) to find a relationship between the $\mathrm{BC}(H)$ and the spectral type. For $\mathrm{M} 7$ and $\mathrm{M} 8$, we find a range of $\mathrm{BC}_{H}$ from 2.54 to 2.78 . Using a solar $M_{\mathrm{bol}}$ of 4.75 , we derive a luminosity for HR

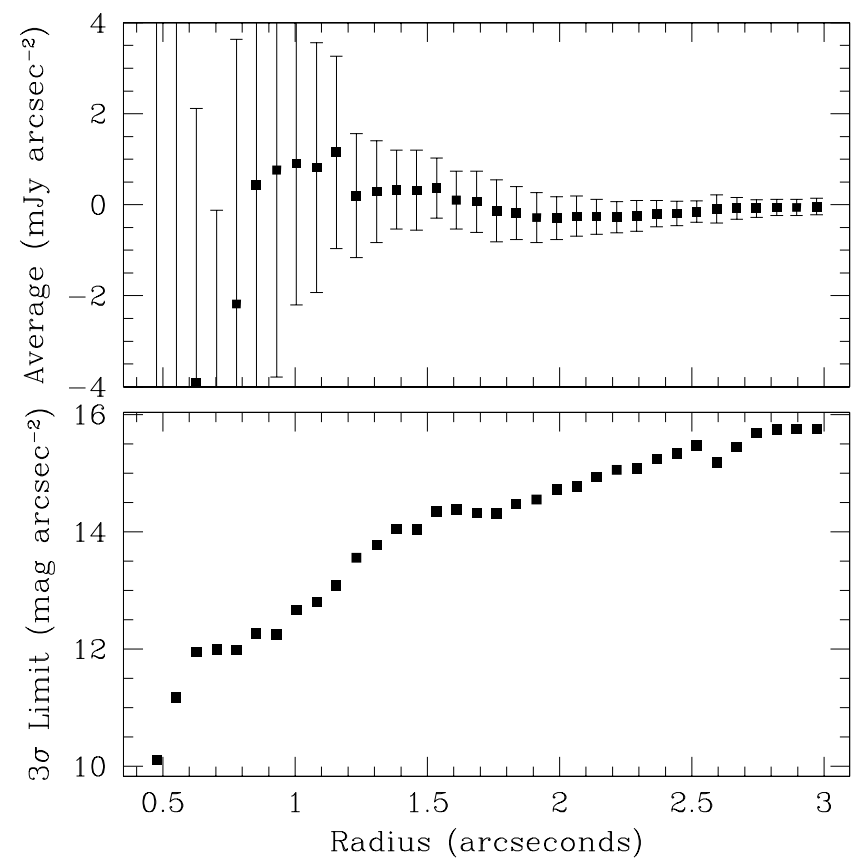

FIG. 5.-Top: a plot of the azimuthally averaged residual surface brightness after subtraction of a coronagraphic PSF. Bottom: $3 \sigma$ limits on detection of disk flux as a function of distance from HR 7329A.
7329B of $0.0026 \pm 0.0003 L_{\odot}$, with an uncertainty that includes the 0.5 spectral type range, the $\mathrm{BC}$, and the distance errors.

\subsection{Derived Mass}

We place HR 7329B on pre-main-sequence evolutionary tracks (Baraffe et al. 1998) to infer a mass (Fig. 4). Assuming only companionship (and therefore distance) indicates a mass of less than $50 M_{\mathrm{Jup}}$ (less than $35 M_{\mathrm{Jup}}$ is not covered in Baraffe's models) and an age of less than $30 \mathrm{Myr}$. This supports the young age attributed to HR 7329A from its position on the H-R diagram, other youth indicators, and possible membership in a young moving group. Evolutionary tracks from different authors do differ somewhat because of the different model atmospheres used. The tracks of D'Antona \& Mazitelli (1997) indicate a mass range of 40 $\mathrm{M}_{\text {Jup }}$ or less for this luminosity and temperature. The models of Burrows et al. (1997) predict that a $40 M_{\text {Jup }}$ brown dwarf will have an effective temperature of $2800 \mathrm{~K}$ and a luminosity of $0.0023 L_{\odot}$ at an age of $22 \mathrm{Myr}$.

\section{LIMITS ON DISK DETECTION}

To look for possible reflected light from a circumstellar disk around the primary, we subtracted an observed coronagraphic PSF from each roll of HR 7329. The NICMOS PSF is time variable, exhibiting small-amplitude structural changes over multiorbit timescales (Kulkarni et al. 2000). To find the best matched coronagraphic PSF to HR 7329, we tested each observation of the 40 other stars in our NICMOS program to see which gave the lowest noise subtraction as measured in the diffraction spikes and in annuli from $0^{\prime \prime} 3$ to $4^{\prime \prime}$. There was no evidence of excess scattered light from a disk in any of the subtractions, but the first visit of the star HD 17925 observed on 1998 September 26 gave the lowest subtraction residuals. This K1 V star is 2.3 times brighter than HR 7329 at F160W. A plot of the azimuthally averaged residual surface brightness after subtraction is shown in the top panel of Figure 5. The error bars represent the standard deviation of all of the pixels (not including pixels obscured by diffraction spikes) averaged at each radius. The residuals are everywhere consistent with zero, i.e., no disk detection. The bottom panel shows these uncertainties multiplied by 3 and converted to F160W magnitudes as a measure of the disk flux that could have been detected at each radius.

HR 7329 appears in the IRAS point source catalog as having excess thermal infrared emission, indicating orbiting dust (Mannings \& Barlow 1998). After color-correcting the catalog fluxes for the spectral index and subtracting the stellar photospheric contribution, the flux densities are $F_{12 \mu \mathrm{m}}=0.25 \pm 0.09 \quad \mathrm{Jy}, \quad F_{25 \mu \mathrm{m}}=0.36 \pm 0.05 \quad \mathrm{Jy}, \quad$ and $F_{60 \mu \mathrm{m}}=0.52 \pm 0.05 \mathrm{Jy}$, with an upper limit of $1 \mathrm{Jy}$ at 100 $\mu \mathrm{m}$. These give a total dust optical depth of $\tau=L_{\mathrm{IR}} / L_{\mathrm{star}} \approx$ $3.5 \times 10^{-4}$, which is an order of magnitude smaller than other similar stars at comparable distances, such as HR 4796 and HD 141569, around which NICMOS imaged the disks (Schneider et al. 1999; Weinberger et al. 1999).

\section{DISCUSSION}

We present high signal-to-noise ratio, near-infrared photometry and optical spectroscopy of a probable companion (HR 7329B) at a projected distance of $200 \mathrm{AU}$ from HR 7329 (A). We suggest that the mass of B is less than $40 M_{\text {Jup }}$. 
The derived age of less than $30 \mathrm{Myr}$ for this companion supports the very young age of the primary A0 V star, which is indicated by its placement on the H-R diagram of nearby A-type stars. We do not detect any $1.6 \mu \mathrm{m}$ scattered light from the far-infrared emitting dust seen by IRAS around HR 7329A.

The HR 7329 system stands out from other binaries in that it has a very high mass ratio, $q \sim 0.01$. Zuckerman \& Becklin (1992) found that among 200 white dwarf stars whose progenitors are $\mathrm{F}$ and $\mathrm{A}$ main-sequence stars, the percentage of systems with low-mass $M$ star companions $\left(M \sim 0.1 M_{\odot}, q \sim 0.06\right)$ was $5 \%-10 \%$ and that the number of detected brown dwarfs was one (GD 165B), which is less than $1 \%$. The small percentage of white dwarfs with detectable brown dwarf companions is probably the result of the decline in brown dwarf luminosity with age. The discovery of the brown dwarf HR 7329B among a small sample of young $\mathrm{A}$ and $\mathrm{F}$ stars $(\sim 10)$ observed by NICMOS suggests that the number of companion brown dwarfs and low-mass stars may not be too different. In the field (Reid 1999) and the Pleiades cluster (Zapatero Osorio et al. 1997), the relative number of low-mass stars and brown dwarfs per log mass interval is also about equal, suggesting a flat initial mass function (IMF) for single stars. Clearly, greater statistics are needed before firm conclusions can be reached about the IMF of secondaries.

We would like to thank M. Jura, C. Chen, and J. Patience for their invaluable help and assistance. We thank the anonymous referee for comments that helped to clarify the presentation. This work is supported in part by NASA grants NAG5-4688 to UCLA and NAG5-3042 to the University of Arizona NICMOS Instrument Definition Team. This paper is based on observations obtained under grant GO-8176.01-97A with the NASA/ESA Hubble Space Telescope at the Space Telescope Science Institute, which is operated by the Association of Universities for Research in Astronomy, Inc., under NASA contract NAS5-26555.

\section{REFERENCES}

Abt, H. A., \& Morrell, N. I. 1995, ApJS, 99, 135

Allard, F., Hauschildt, P. H., Alexander, D. R., \& Starrfield, S. 1997, ARÄ\&A, 35, 137

Baraffe, I., Chabrier, G., Allard, F., \& Hauschildt, P. H. 1998, A\&A, 337, 403

Barrado y Navascues, D., Stauffer, J. R., Song, I., \& Caillult, J.-P. 1999, ApJ, 520, L123

Bessel, M. S., Castelli, F., \& Plez, B. 1998, A\&A, 333, 231

Burrows, A., et al. 1997, ApJ, 491, 856

D'Antona, F., \& Mazzitelli, I. 1997, Mem. Soc. Astron. Italiana, 68, 807

Henry, T. 1991, Ph.D. thesis, Univ. Arizona

Jura, M., Malkan, M., White, R., Telesco, C., Pina, R., \& Fisher, R. S. 1998, ApJ, 505, 897

Kirkpatrick, J. D., Henry, T. \& Irwin, M. J. 1997, AJ, 113, 1421

Kirkpatrick, J. D., Henry, T., \& McCarthy, D. W. 1991, ApJS, 77, 417

Kirkpatrick, J. D., Kelly, D. M., Rieke, G. H., Liebert, J., Allard, F., \& Wehrse, R. 1993, ApJ, 402, 643

Kirkpatrick, J. D., \& McCarthy, D. W. 1994, AJ, 107, 333

Kulkarni, V. P., Hill, J. M., Schneider, G., Weymann, R. J., StorrieLombardi, L. J., Rieke, M. J., Thompson, R. I., \& Januzzi, B. 2000, ApJ, 536,36

Leggett, S. K., Allard, F., Berriman, G., Dahn, C. C., \& Hauschildt, P. H. 1996, ApJS, 104, 117

Leggett, S. K., Allard, F., \& Hauschildt, P. H. 1998, ApJ, 509, 836

Lowrance, P. J., et al. 1999, ApJ, 512, L69

Luhman, K. L., \& Rieke, G. H. 1998, ApJ, 497, 354

MacKenty, J. W., et al. 1997, NICMOS Instrument Handbook, Version 2.0, (Baltimore: STScI)

Mannings, V., \& Barlow, M. J. 1998, ApJ, 497, 330

Reid, N. 1999, in Proc. Star Formations, ed. T. Nakamoto. (Nobeyama: NRO)

Schneider, G., et al. 1999, ApJ, 513, L127

Schneider, G., Thompson, R. I., Smith, B. A., \& Terrile, R. J. 1998, Proc. SPIE, 3356, 222

Stauffer, J. R., Hartmann, L. W., \& Barrado y Navascues, D. 1995, ApJ, 454,910

Tinney, C. G., Mould, J. R., \& Reid, I. N. 1993, AJ, 105, 1045

Weinberger, A. J., Becklin, E., Schneider, G., Smith, B. A., Lowrance, P. J., Silverstone, M., Zuckerman, B., \& Terrile, R. 1999, ApJ, 535, L53

Weinberger, A. J., Rich, R. M., Becklin, E. E., Zuckerman, B., \& Matthews, K. 2000, ApJ, submitted

Zapatero Osorio, M. R., Rebolo, R., Martin, E. L., Basri, G., Magazzu, A., Hodgkin, S. T., Jameson, R. F., \& Cossburn, M. R. 1997, ApJ, 491, L81

Zuckerman, B., \& Becklin, E. E. 1992, ApJ, 386, 260

Zuckerman, B., \& Webb, R. A. 2000, ApJ, 535, 959 